Aim of the study: Intraoperative radiotherapy (IORT) may improve outcome of surgical treatment of recurrent colorectal cancer (CRC). The aim of this study is to determine the feasibility, safety and long-term results of surgical treatment of recurrent CRC with orthovolt IORT.

Material and methods: Fifty-nine consecutive CRC patients with local recurrence (LR), undergoing surgery, were included in the retrospective analysis of prospectively collected data. The modified Wanebo classification was used to stage LR (Tr). Twenty-five (43\%) patients received IORT using INTRABEAM ${ }^{\oplus}$ PRS 500. The complications were classified according to the Clavien-Dindo classification.

Results: There were 32 males and 27 females, with a median age of 63 years. Multi-visceral resections were performed in 37 (63\%) patients. Median hospitalization time after surgery with IORT was 7 days. One (1.7\%) in-hospital postoperative death was reported. Grade 3/4 postoperative complications were found in $11(19 \%)$ patients. Intraoperative radiotherapy had no effect on the postoperative hospitalization time, morbidity and mortality. Median survival after RO resection was 32 months. Complete resection (RO), no synchronous liver metastases (MO), and no lateral and posterior pelvic wall involvement, were significant predictors of improved survival. Stage of LR was found to be an independent prognostic factor in the multivariate analysis ( $p=0.03)$; Cox regression model). In patients with $L R$ stage < Tr5, a 3-year overall survival (OS) rate was $52 \%$.

Conclusions: Combination of surgical resection and orthovolt IORT is a safe and feasible procedure that does not increase the risk of postoperative complications or prolongs the hospital stay. Despite aggressive surgery supported by IORT, the advanced stage of LR is a limiting factor of long-term survival.

Key words: colorectal cancer, recurrence, intraoperative radiotherapy, surgery, survival.

Contemp Oncol (Pozn) 2016; 20 (1): 52-57 DOI: $10.5114 /$ wo.2016.58500

\section{Intraoperative radiotherapy with low energy photons in recurrent colorectal cancer: a single centre retrospective study}

\author{
Magdalena Skórzewska, Jerzy Mielko, Andrzej Kurylcio, Jarosław Romanek, \\ Wojciech P. Polkowski
}

Department of Surgical Oncology of the Medical University of Lublin, Lublin, Poland

\section{Introduction}

In patients with local recurrence (LR) of colorectal cancer (CRC), the potential for a positive resection margin remains high and may have a negative impact on overall survival (OS) [1, 2]. Improved local control and OS rates have been reported when intraoperative radiotherapy (IORT) was used after neoadjuvant chemoradiotherapy for locally recurrent disease in radiation-naïve patients [3]. Besides re-irradiation from external fields, the IORT application is the only option for LR in patients after radiotherapy used for the primary tumour [4]. The only available data for the IORT come from retrospective, single-institution studies [3, 5]. Based on the available literature, national and international reports, AIRO IORT Study Group considered the pelvic recurrence of CRC as the indication for the use of IORT. Surgical resection alone of CRC recurrence is frequently associated with a high risk of residual tumor tissue in the tumour bed [6]. The principle for the use of IORT is to eliminate microscopic tumour foci by maximizing the radiobiological effects of a single dose of radiation and to optimize the treatment duration [7, 8]. The advantage of IORT over the external beam radiotherapy (EBRT) boost is the possibility of administering high dose of radiation while dose-limiting structures, such as the bowel, bladder or ureters, are safely shielded [4]. Moreover, during surgery it is possible to release adhesions, moving normal tissues beyond the irradiation field, thereby protecting them while giving an appropriate dose to the precisely defined surgical bed area with a safe margin $[5,9]$. The main advantage of IORT is sterilizing close or positive resection margins [10-12].

Treatment of CRC recurrence is an issue reluctantly undertaken in scientific research, which is also reflected in everyday clinical practice. Due to sparse literature data on the use of orthovolt IORT in the treatment of recurrent CRC, we found it relevant to present the review of our clinical experience in this field. Therefore, the aim of this study was the assessment of early and late results of surgical treatment combined with IORT with low-energy photons in patients with recurrent CRC.

\section{Material and methods}

This is a retrospective analysis of prospectively collected clinical data on patients treated for locally recurrent CRC between January 2004 and December 2011 at the Department of Surgical Oncology of the Medical University of Lublin.

\section{Patient selection}

Qualification for treatment was undertaken individually for each patient upon the opinion of a multidisciplinary team. The decision was based on patients' general condition, signs of resectability on computed tomography/ 
magnetic resonance (CT/MR) imaging and prior use of radiotherapy. Patients eligible for surgical resection were divided according to the perioperative risk into low/moderate or high surgical risk groups. In the first group of patients, radical, multivisceral en-bloc resections were planned with a possibility of IORT applied via laparotomy and/or the perineal/sacral wound. In high risk patients, provided that the localization of recurrent CRC was intraluminal (anastomotic), local transanal excision was performed with the Parks method using Transanal Microsurgical Tumour resection instrumentation (TMT; B. Braun Aesculap, Melsungen, Germany). In these few patients, IORT was also applied transanally in the manner similar to the contact X-ray therapy [13].

\section{Radiotherapy use}

Patients were qualified for surgical resection with $\mathrm{cu}$ rative intent and IORT. Synchronous (with LR), resectable liver metastases were not contraindication for surgery and IORT. Intraoperative radiotherapy was applied only to the tumour bed after resection, while dose-limiting structures such as the small bowel or ureters were moved from the irradiation field and protected. Intraoperative radiotherapy was performed using a dedicated INTRABEAM ${ }^{\oplus}$ PRS 500 (Photon Photoelectron Radiosurgery System Corporation, Carl Zeiss, Oberkochen, Germany). Operation of the INTRABEAM system is based on the use of a directed, orthovolt X-ray beam (photons with an energy of maximum $50 \mathrm{keV}$ ). Spherical shape applicators with diameters ranging from $1.5 \mathrm{~cm}$ to $5.0 \mathrm{~cm}$ allow precise and uniform dose distribution on the surface of the post-resection lodge. The radiation generated by the system is rapidly attenuated in the soft tissues according to the inverse cubic law $\left(1 / r^{3}\right)$. This reduces the exposure of normal, surrounding tissues and critical organs and minimizes the need for radiation protection of medical personnel. The radiation dose was prescribed to the surface of the applicator attached in vivo to the tissue of the resection margin. The irradiation time varies depending on the radiation dose and the diameter of the applicator used.

\section{Stage of the recurrent tumour}

The stage of LR of rectal cancer in this study was defined by two classification systems: Wanebo and Suzuki-Gunderson, both based on intraoperative macroscopic tumor features and microscopic evaluation of surgical specimens $[14,15]$. For the purpose of this study, the original four-grade Wanebo classification was modified into three stages, where the infiltration of full thickness of the intestinal wall comprised one stage sequence $\left(\operatorname{Tr}_{1-3}\right)$. The remaining grades were identical with the original classification [15]. The Suzuki-Gunderson classification describes four stages of immobilization of the tumor relative to pelvic structures by determining the number of locations with direct invasion $[16,17]$.

\section{Completeness of resection}

Completeness of resection was assessed using a standard residual disease (R) classification, according to the Union for International Cancer Control (UICC) [16]. The ex- tent of the surgery was dependent on the age and general condition of the patient, location of LR, co-morbidities and the extent of primary tumour resection. In patients with recurrent tumour directly infiltrating the adjacent organs, extended, multivisceral, en-bloc resections were performed. Ureteral stents were placed selectively before surgery only when collision with the urinary system (on preoperative imaging) was suspected. Radicality of resection was evaluable in 57 of 59 patients. The surgical complications were scored according to the Clavien-Dindo classification [18]. Only major surgical complications, grade 3 (requiring surgical intervention), grade 4 (life-threatening), and grade 5 (death of a patient) were reported.

\section{Statistical analysis}

Statistical analyses were performed using SPSS Statistical Software for Windows version 17.0.2. (SPSS Inc. Chicago, IL). Survival rates were estimated using the Kaplan-Meier curve, and comparisons of survival between groups were made using the log-rank method. Multivariate analysis was performed using the Cox proportional-hazard method. Results of comparisons between surgery with IORT and surgery alone group in terms of postoperative hospitalisation stay and surgical complications were correlated using non-parametric methods (Mann-Whitney $U$-test and the Chi-square test, respectively). P-value of $<0.05$ was regarded as statistically significant.

\section{Results}

The analysis included 59 patients, who underwent surgical resection of LR, 32 women and 27 men, aged from 26 to 77 years (median 63 years). Eleven patients underwent resection for recurrent colon cancer and 48 patients for rectal cancer. The median time from the first diagnosis of CRC to current treatment of LR was 24 months, assessed in 48 (81\%) patients (range: 6-134 months). The follow-up time ranged from 1 to 57 months. The median follow-up time for the entire study group was 11 months and 20 months in surviving patients. Seven of 59 patients (12\%) were lost to follow-up due to not reporting for checkups in the Outpatient Clinic. During the follow-up period 35 (59\%) patients died.

In patients undergoing resection the following surgical procedures were performed: left-sided hemicolectomy $(n=4)$, excision of the recurrent tumour $(n=18)$, Hartmann's resection $(n=6)$, abdomino-sacral amputation of the rectum $(n=14)$, anterior resection of the rectum $(n=9)$, local transanal excision (TMT) $(n=6)$, and local excision of skin/soft tissue recurrences in postoperative scar $(n=1)$ and colostomy area $(n=1)$. Extended resection was performed in 37 out of 59 (63\%) patients, and in 5 (8\%) cases resection involved more than one organ. Extended resection supplemented with IORT was performed in 16 (64\%) patients.

Intraoperative radiotherapy was performed in $25 \mathrm{pa}$ tients, including 3 patients with recurrent colon cancer and 22 patients with recurrent rectal cancer. The median radiation surface dose was 12.5 Gy (range: 10-17.5 Gy). The median applicator size used was $4 \mathrm{~cm}$ (range: $2-5 \mathrm{~cm}$ ). All 
Table 1. Treatment characteristics of patients with recurrent CRC who underwent resection with IORT

$\begin{array}{lcc}\text { Resection type } & \text { CR }(n=3) & \text { RR }(n=22) \\ \text { tumour excision } & 2 & 7 \\ \text { left hemicolectomy } & 1 & - \\ \text { ASAR } & - & 3 \\ \text { hartmann resection } & - & 3 \\ \text { LAR } & - & 3 \\ \text { TMT } & - & 6 \\ & \text { IORT } & \\ \begin{array}{l}\text { Dose (Gy) } \\ \text { Me, range }\end{array} & 15 ; 12.5-15 & 12.5 ; 10-17.5 \\ \text { Radiation time (minutes) } & & \\ \text { Me, range } & 21 ; 14-31 & 17 ; 6-47\end{array}$

$C R$ - recurrent colon cancer. $R R$ - recurrent rectal cancer. $A S A R$ - abdominosacral amputation of the rectum; $L A R$ - low anterior resection of the rectum TMT - transanal microsurgical tumour excision; IORT - intraoperative radiation therapy; Gy -Grey; $n$ - number of patients

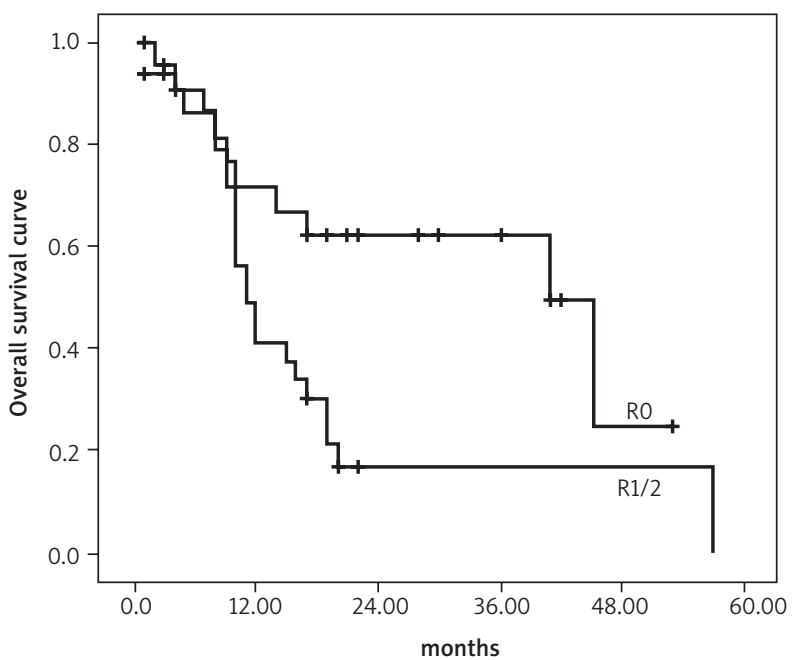

Fig. 1. Survival curves of patients with recurrent CRC according to radicality of resection (RO vs. $\mathrm{R} 1 / 2$ )

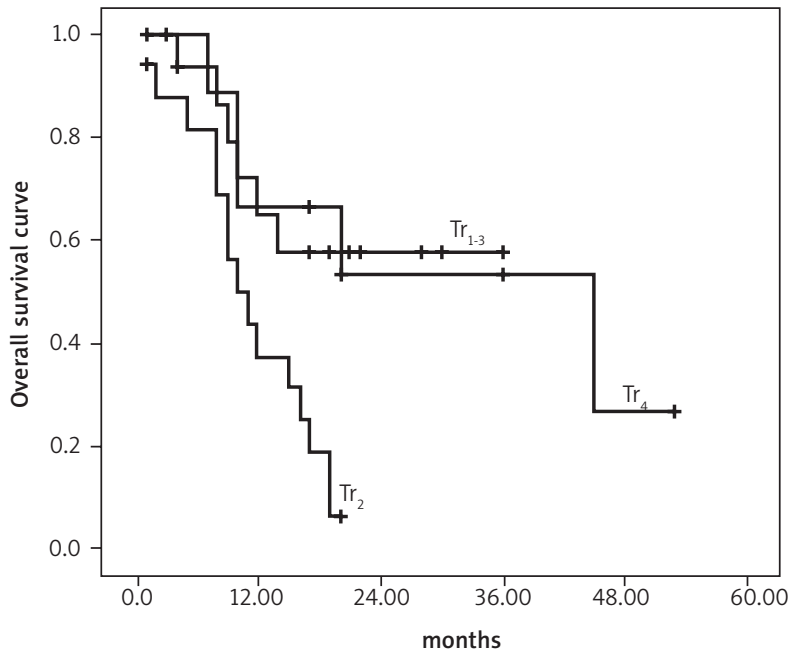

Fig. 2. Survival curves of patients with recurrent CRC in various stages of recurrent tumour according to the modified Wanebo classification (Tr1-3 vs. Tr4 vs. Tr5)
Table 2. Early postoperative outcome in patients after surgery with IORT and after surgery alone

$\begin{array}{lcc}\text { Postoperative } & \begin{array}{c}\text { Surgery with IORT } \\ (n=25)\end{array} & \begin{array}{c}\text { Surgery alone } \\ (n=34)\end{array} \\ \begin{array}{l}\text { Hospitalisation time (days) } \\ \text { Me; range } \\ \text { (without TMT) }\end{array} & 10 ; 4-23 & 8 ; 2-22 \\ \text { TMT (days) Me; range } & 3 ; 2-5 & \\ \text { Complications } n(\%) & 4(16) & 7(21) \\ \text { TMT } & 0 & \\ \text { 30-day mortality } n(\%) & 0 & 0 \\ \text { 90-day mortality } n(\%) & 0 & 1(1.7)\end{array}$

IORT(+) - surgery with IORT; IORT(-) - surgery alone; $n$ - number of patients; Me - median; TMT - transanal microsurgical tumour resection; values in brackets are percentages

sites irradiated with IORT, were smaller than $5 \mathrm{~cm}$ in size. The median duration of IORT was 18 minutes (range: 6-47 minutes).

Comparison of treatment characteristics in patients with recurrent $C R C$ treated with resection combined with IORT is summarized in Table 1.

The median survival time in patients who underwent resection with IORT was 24.0 months, whereas in patients with resection alone was 24.6 months ( $p=0.891$, log-rank test). The three-year overall survival rate after surgery and IORT was 31\% while after surgery alone $37 \%$.

The median discharge time after surgery in patients with recurrent colon cancer was 9 days (range 6-22) and in patients with recurrent rectal cancer 8 days (range 2-23). No intraoperative complications were attributed to IORT. One (1.7\%) postoperative death (grade $5 \mathrm{com}$ plication) was reported. A female patient after extensive surgery alone died on postoperative day 114, after five re-laparotomies because of recurrent intestinal fistula. The cause of death was septicemia in the course of diffuse purulent peritonitis. No significant differences were found in the time of hospitalization ( $p=0.238$; Mann-Whitney $U$ test) and incidence of postoperative complications ( $p=$ $=0.654 ; \chi^{2}$ test) between patients after surgery with IORT and surgery alone (Table 2).

In the entire group of patients $\mathrm{RO}$ resection was performed in 25 (44\%) patients, R1 in 11 (19\%) and R2 in 21 (37\%). In patients who underwent resection with IORT, RO, $\mathrm{R} 1$ and $\mathrm{R} 2$ resection rates were $32 \%, 24 \%$ and $44 \%$, respectively. The median survival time of patients after $\mathrm{RO}$ resection was 32.7 months whereas after non-radical resection R1/2 was only 19.1 months. The observed difference was statistically significant ( $p=0.018$, log-rank test; Fig. 1 ).

Twelve (20\%) of 59 patients underwent simultaneous hepatic resection for synchronous metastases to the liver at the time of surgery for LR. The median survival time of patients with liver metastases was only 11 months. Median overall survival of patients with and without liver metastases was 11.1 and 27.1 months, respectively ( $p=0.035$, log-rank test).

Stage $\operatorname{Tr}_{1-3}$ of the recurrent tumour according to the modified Wanebo classification was found in 19 (41\%) patients, $\mathrm{Tr}_{4}$ in 10 (22\%), and $\operatorname{Tr}_{5}$ in 17 (37\%) patients. The median 
survival time of patients with $\operatorname{Tr}_{4}$ was 31.8 months, with $\operatorname{Tr}_{1-3}$ - 24.8 months and with $\operatorname{Tr}_{5}$ only 11.4 months. The observed difference was statistically significant $(p=0.006$, log-rank test; Fig. 2).

On univariate survival analysis, none of the following prognostic factors was found to be associated with longterm survival: gender, age, time interval to recurrence, extent of resection, location of recurrence, IORT dose and Suzuki-Gunderson staging system. Otherwise, the modified Wanebo classification (nominal variable: $\operatorname{Tr}_{1-4}$ vs. $\operatorname{Tr}_{5}$ ), radicality of resection (nominal variable: RO vs. R1/2) and liver metastases (nominal variable: MO vs. M1) were found significant prognosticators.

Multivariate analysis of the three significant variables that emerged from the univariate analysis (modified Wanebo classification, radicality of resection and presence of liver metastases), revealed that only the stage of LR tumour independently influenced long-term survival (Table 3). Median survival time in patients with $\operatorname{Tr}_{1-4}$ LR was 32 months and 3-year overall survival was 52\%.

\section{Discussion}

A significant increase in long term survival of patients with recurrent CRC after IORT has been reported [17, 19, 20]. Radical (RO) resection of $L R$ is associated with at least twice higher survival than in non-radical (R1/R2) resection and remains the treatment of choice [21, 22]. The tumor-free resection margin is a favorable prognostic factor for improved local control and overall survival [19-21, 23-26]. In our study the survival time of patients after RO resection was longer in comparison to non-radical resection group. This finding is in agreement with the results of meta-analysis by Bhangu et al. who found no survival benefit of macroscopically non-radical (R2) resection over non- resection (laparotomy) [21].

Intraoperative radiotherapy allows the administration of a single, high dose of radiation applied during surgery under direct vision $[27,28]$. Although the use of IORT as a component of combined multimodal therapy may increase the survival by improving the local control, there are no randomized trials on IORT in CRC $[9,25]$. Meta-analysis of the non-randomized reports in the treatment of recurrent CRC showed improved 5-year overall survival after IORT [29]. Moreover, better local control with a lower risk of relapse at higher doses of radiation have been reported with the use of IORT [5, 11]. There are only two reports describing the use of orthovolt IORT in CRC recurrences [10, 23, 40].

Local recurrence of CRC located in the pelvic cavity is associated with a high risk of infiltration of the surrounding bony structures, which drastically reduces the chances of radical resection that usually involves the extended resections of multiple organs [30, 31]. Although extended multivisceral resections were performed in the majority of cases (84\%) in this study, no differences in the survival depending on the extent of resection were found, which is consistent with other reports [17, 32, 33].

In our study, only serious postoperative complications requiring re-laparotomy were evaluated. No increase in the complication rate with extended resection was found. In
Table 3. Multivariate analysis of prognostic factors

\begin{tabular}{lcccc} 
Prognostic factor & $p$ & $\operatorname{Exp}(\mathrm{B})$ & \multicolumn{2}{c}{$\% \mathrm{Cl}$ for Exp (B) } \\
\cline { 4 - 5 } & & & lower limit & upper limit \\
$\begin{array}{l}\text { modified } \\
\begin{array}{l}\text { Wanebo } \\
\text { classification }\end{array}\end{array}$ & 0.030 & 3.59 & 1.13 & 11.33 \\
$\begin{array}{l}\text { resection } \\
\text { radicality }\end{array}$ & 0.793 & 0.84 & 0.23 & 3.04 \\
$\begin{array}{l}\text { distant (liver) } \\
\text { metastases }\end{array}$ & 0.219 & 1.80 & 0.71 & 4.57
\end{tabular}

$64 \%$ of cases after $\mathrm{R} 2$ resection, severe post-surgical complications occurred. Higher postoperative complications rate was found in patients with recurrent rectal carcinoma (21\%) compared to patients with recurrent colon cancer (9\%). This difference may be attributable to the fact that surgical resection in the group of rectal cancer recurrence is associated with much greater technical difficulties.

In the majority of studies, distant metastases are the criterion for exclusion from the study. However in the present study, $8 \%$ of patients who underwent simultaneous resection of liver metastases were included and lived significantly shorter (11 months) than patients with local disease (27 months). Bhangu et al. also obtained lower 3-year survival rates in patients with distant metastases (33\% vs. 54\%) [22].

Moore et al. reported that the $\mathrm{RO}$ resection rate was significantly higher when LR was located axially/centrally or anteriorly in the pelvis [34]. Infiltration of pelvic side walls accounted for a lower radical resections rate $(60 \%$ vs. 19\%) [34]. However, the complication rate after extended resection was found to be higher (32\%) as compared to standard resection (21\%) [32]. In our analysis, the staging of rectal cancer recurrence was assessed according to the modified Wanebo classification. The infiltration of side walls is associated with worse outcomes and obtaining radical resection is then significantly reduced [24, 35]. Median survival in patients with $\mathrm{MO}$, RO resection with IORT and in stage $\operatorname{Tr}_{1-4}$ of the Wanebo classification was 26 months.

Radiation-induced toxicity is extremely difficult to distinguish from the surgical complication or symptoms of disease progression [36]. The most common types of early postoperative complications are those associated with wound healing $3-46 \%$ and pelvic abscesses, intestinal obstructions and fistulas [17, 20, 22, 29, 32, 37]. Postoperative complications vary from $15 \%$ to $68 \%$ [38]. The percentage of serious postoperative complications ranges from $27 \%$ to $81 \%[20,21,28,35]$. The most common complication after IO(E)RT is small bowel obstruction (14\%) and pelvic abscesses $(12 \%)$ [17, 20]. Intraoperative radiotherapy is a technique that does not seem to increase the rate of complications or mortality rates [37]. Postoperative mortality associated with the use of IORT ranges from 0 to $3.6 \%$, irrespective of the IORT used [10, 23, 32]. In the literature, 3-month postoperative mortality rates range from $0 \%$ to $8 \%[4,26,28,29,37,39]$. In the present study, two 
postoperative deaths were reported (2.5\%); however, none of them was attributed to the use of IORT.

In the present study we demonstrated that RO resection should be the treatment of choice, the presence of (even resectable) liver metastases is associated with poor prognosis, and infiltration of the lateral and posterior compartments of the pelvis is associated with significantly shorter survival. Our experience with the use of orthovolt IORT highlights the need for better strategies of combined therapy and multidisciplinary care for patients with recurrent CRC. Unsatisfactory results in patients treated with IORT arise mostly from the late diagnosis of the LR and inability to obtain the free resection margin $[36,40]$.

\section{Conclusions}

Combination of surgical resection and orthovolt IORT is a safe and feasible procedure that does not increase the risk of postoperative complications or prolongs the hospital stay.

The macroscopically non-radical (R2) resection of the $L R$, even if compensated by orthovolt IORT, is not justified, as the results do not support the hypothesis of the added value of IORT.

Despite aggressive surgery (multivisceral including hepatic resections) supported by orthovol IORT, the advanced stage of $L R$ is a limiting factor of long-term survival.

The authors would like to thank drs Matgorzata Jankiewicz and Beata Winkler from St. John's Cancer Center (Lublin, Poland), for performing IORT procedures.

Wojciech P. Polkowski has received travel and speaker honorarium funds from Zeiss. The authors have no other relevant affiliations or financial involvement with any organization or entity with a financial interest in or financial conflict with the subject matter or materials discussed in the manuscript.

Magdalena Skórzewska, Jerzy Mielko, Andrzej Kurylcio, Jarostaw Romanek declare no conflict of interest.

\section{References}

1. Ferenschild FT, Vermaas M, Nuyttens JJ, et al. Value of intraoperative radiotherapy in locally advanced rectal cancer. Dis Colon Rectum 2006; 49: 1257-65.

2. Vermaas M, Ferenschild FT, Nuyttens JJ, et al. Preoperative radiotherapy improves outcome in recurrent rectal cancer. Dis Colon Rectum 2005; 48: 918-28.

3. Willett CG, Czito BG, Tyler DS. Intraoperative radiation therapy. J Clin Oncol 2007; 25: 971-7.

4. Roeder F, Goetz JM, Habl G, et al. Intraoperative Electron Radiation Therapy (IOERT) in the management of locally recurrent rectal cancer. BMC Cancer 2012; 12: 592.

5. Yeung JM, Ngan S, Lynch C, Heriot AG. Intraoperative radiotherapy and colorectal cancer. Minerva Chir 2010; 65: 161-71.

6. Valentini V, Impiombato FA, De Paoli A, Fortuna G, Tomio L, Mantini $G$. The use of intraoperative radiation therapy according to evidence-based medicine. Tumori 2005; 4: 64-74.

7. Eble MJ, Lehnert T, Treiber M, Latz D, Herfarth C, Wannenmacher M. Moderate dose intraoperative and external beam radiotherapy for locally recurrent rectal carcinoma. Radiother Oncol 1998; 49: 169-74.
8. Calvo FA, Meirino RM, Orecchia R. Intraoperative radiation therapy first part: rationale and techniques. Crit Rev Oncol Hematol 2006; 59: 106-15.

9. Bouchard P, Efron J. Management of recurrent rectal cancer. Ann Surg Oncol 2010; 17: 1343-56.

10. Guo S, Reddy CA, Kolar M, et al. Intraoperative radiation therapy (IORT) with the photon radiosurgery system (PRS) in locally advanced and recurrent rectal cancer: retrospective review of the Cleveland clinic experience. Radiat Oncol 2012; 7: 110.

11. Rodriguez-Bigas MA, Chang GJ, Skibber JM. Multidisciplinary approach to recurrent/unresectable rectal cancer: how to prepare for the extent of resection. Surg Oncol Clin N Am 2010; 19: 847-59.

12. Merrick HW 3rd, Dobelbower RR Jr. Intraoperative radiation therapy in surgical oncology. Surg Oncol Clin N Am 2003; 12: 883-97.

13. Gérard JP, Myint AS, Croce O, Lindegaard J, Jensen A, Myerson R, Hannoun-Lévi JM, Marcie S. Renaissance of contact X-ray therapy for treating rectal cancer. Expert Rev Med Devices 2011; 8: 483-92.

14. Wanebo HJ, Koness RJ, Vezeridis MP, Cohen SI, Wrobleski DE. Pelvic resection of recurrent rectal cancer. Ann Surg 1994; 220: 586-97.

15. Wanebo HJ, Antoniuk P, Koness RJ, Levy A, Vezeridis M, Cohen SI, Wrobleski DE. Pelvic resection of recurrent rectal cancer: technical considerations and outcomes. Dis Colon Rectum 1999; 42: 1438-48.

16. Suzuki K, Dozois RR, Devine RM, Nelson H, Weaver AL, Gunderson LL, Ilstrup DM. Curative reoperation for locally recurrent rectal cancer. Dis Colon Rectum 1996; 39: 730-6.

17. Suzuki K, Gunderson LL, Devine RM, Weaver AL, Dozois RR, Ilstrup DM, Martenson JA, O'Connell MJ. Intraoperative irradiation after palliative surgery for locally recurrent rectal cancer. Cancer 1995; 75: 939-52.

18. Clavien PA, Barkun J, de Oliveira ML, et al. The Clavien-Dindo classification of surgical complications: five-year experience. Ann Surg 2009; 250: 187-96.

19. Calvo FA, Meirino RM, Orecchia R. Intraoperative radiation therapy part 2. Clinical results. Crit Rev Oncol Hematol 2006; 59: 116-27.

20. Hashiguchi Y, Sekine T, Sakamoto H, et al. Intraoperative irradiation after surgery for locally recurrent rectal cancer. Dis Colon Rectum 1999; 42: 886-93.

21. Bhangu A, Ali SM, Darzi A, Brown G, Tekkis P. Meta-analysis of survival based on resection margin status following surgery for recurrent rectal cancer. Colorectal Dis 2012; 14: 1457-66.

22. Bhangu A, Ali SM, Cunningham D, Brown G, Tekkis P. Comparison of long-term survival outcome of operative vs nonoperative management of recurrent rectal cancer. Colorectal Dis 2013; 15: 156-63.

23. Daly ME, Kapp DS, Maxim PG, Welton ML, Tran PT, Koong AC, Chang DT. Orthovoltage intraoperative radiotherapy for locally advanced and recurrent colorectal cancer. Dis Colon Rectum 2012; 55: 695-702

24. Mirnezami AH, Sagar PM, Kavanagh D, Witherspoon P, Lee P, Winter D. Clinical algorithms for the surgical management of locally recurrent rectal cancer. Dis Colon Rectum 2010; 53: 1248-57.

25. Alektiar KM, Zelefsky MJ, Paty PB, Guillem J, Saltz LB, Cohen AM, Minsky BD. High-dose-rate intraoperative brachytherapy for recurrent colorectal cancer. Int J Radiat Oncol Biol Phys 2000; 48: 219-26.

26. Rutten HJ, Mannaerts GH, Martijn H, Wiggers T. Intraoperative radiotherapy for locally recurrent rectal cancer in The Netherlands. Eur J Surg Oncol 2000; 26 Suppl A: 16-20.

27. Zhao J, Du CZ, Sun YS, Gu J. Patterns and prognosis of locally recurrent rectal cancer following multidisciplinary treatment. World J Gastroenterol 2012; 18: 7015-20.

28. Dresen RC, Gosens MJ, Martijn H, et al. Radical resection after IORT-containing multimodality treatment is the most important determinant for outcome in patients treated for locally recurrent rectal cancer. Ann Surg Oncol 2008; 15: 1937-47.

29. Mirnezami R, Chang GJ, Das P, Chandrakumaran K, Tekkis P, Darzi A Mirnezami $\mathrm{AH}$. Intraoperative radiotherapy in colorectal cancer: Systematic review and meta-analysis of techniques, long-term outcomes, and complications. Surg Oncol 2013; 22: 22-35.

30. Dassanayake BK, Samita S, Deen RY, Wickramasinghe NS, Hewavisenthi J, Deen KI. Local recurrence of rectal cancer in patients not receiving neoadjuvant therapy - the importance of resection margins. Ceylon Med J 2011; 56: 159-61. 
31. Hellinger MD, Santiago CA. Reoperation for recurrent colorectal cancer. Clin Colon Rectal Surg 2006; 19: 228-36.

32. Hahnloser D, Nelson H, Gunderson LL, et al. Curative potential of multimodality therapy for locally recurrent rectal cancer. Ann Surg 2003; 237: 502-8.

33. Williams CP, Reynolds HL, Delaney CP, Champagne B, Obias V, Joh YG, Merlino J, Kinsella TJ. Clinical results of intraoperative radiation therapy for patients with locally recurrent and advanced tumors having colorectal involvement. Am J Surg 2008; 195: 405-9.

34. Moore HG, Shoup M, Riedel E, et al. Colorectal cancer pelvic recurrences: determinants of resectability. Dis Colon Rectum 2004; 47: 1599-606.

35. Nag S, Martinez-Monge R, Martin EW. Intraoperative electron beam radiotherapy in recurrent colorectal carcinoma. J Surg Oncol 1999; 72: 66-71.

36. Konski AA, Suh WW, Herman JM, et al. ACR Appropriateness Criteria-Recurrent Rectal Cancer. Gastrointest Cancer Res 2012; 5: 3-12.

37. Wiig JN, Tveit KM, Poulsen JP, Olsen DR, Giercksky KE. Preoperative irradiation and surgery for recurrent rectal cancer. Will intraoperative radiotherapy (IORT) be of additional benefit? A prospective study. Radiother Oncol 2002; 62: 207-13.

38. Nielsen MB, Laurberg S, Holm T. Current management of locally recurrent rectal cancer. Colorectal Dis 2011; 13: 732-42.

39. Turley RS, Czito BG, Haney JC, Tyler DS, Mantyh CR, Migaly J. Intraoperative pelvic brachytherapy for treatment of locally advanced or recurrent colorectal cancer. Tech Coloproctol 2013; 17: 95-100.

40. Skórzewska M, Polkowski WP. Evidence behind use of orthovolt radiotherapy and other techniques of IORT in recurrent colorectal cancer treatment. Transl Cancer Res 2014; 3: 530-6.

\section{Address for correspondence}

Prof. Wojciech P. Polkowski, MD, PhD

Department of Surgical Oncology

Medical University of Lublin

Staszica 11

20-081 Lublin, Poland

tel. +48 815344313

$\mathrm{fax}+48815322395$

e-mail:wojciech.polkowski@umlub.pl

Submitted: 3.07 .2015

Accepted: 29.10.2015 